\title{
De l'inventeur héroïque aux innovations collectives : la ganterie grenobloise en quête perpétuelle du progrès (1830-1930)
}

\author{
From heroic inventors to collective innovations: The Grenoble glove \\ industry in the perpetual search of progress (1830-1930)
}

\author{
Audrey Colonel ${ }^{1}$ \\ ${ }^{1}$ Laboratoire de Recherche Historique Rhône-Alpes, Université Grenoble-Alpes, France, audreycolonel@hotmail.fr
}

RÉSUMÉ. Considérée comme «inventions révolutionnaires » de l'industrie du gant, la main-de-fer et la création de pointures de mains dans les années 1830 ont apporté une grande renommée à Xavier Jouvin (1801-1844), inventeur héroïsé qui suscite toute une littérature dès la fin du XIXe siècle, faisant de l'ombre aux innovations introduites dans la branche à l'initiative de Maisons de ganterie dès le dernier quart du XIXe siècle.

Au début du XXe siècle, la recherche d'innovations dans la gamme de produits mis sur le marché s'intensifie à l'heure où le gant de peau, devenu banal, n'attire plus autant qu'au siècle précédent. Des entreprises de ganterie se dotent de laboratoires de recherches et de bureaux d'études afin de favoriser le progrès technique. Elles intègrent à leurs effectifs chimistes et ingénieurs, à l'image des époux Szmukler travaillant pour les Établissements Reynier dans les années 1920, identifiables sur les brevets et dans la presse technique.

ABSTRACT. Considered "revolutionary inventions" of the glove industry, the iron fist and the creation of hand sizes in the 1830s brought great fame to Xavier Jouvin (1801-1844). He was a heroic inventor who has inspired a great deal of literature since the end of the 19th century, overshadowing the innovations introduced to the industryby glove manufacturers in search of technical progress during the last quarter of the 19th century.

At the beginning of the 20th century, the search for innovations in the product range on the market intensified at a time when the skin glove - which had become commonplace - was no longer as attractive as in the previous century. Glove-making companies were setting up research laboratories and design offices to promote technical progress. They integrated chemists and engineers into their workforce, like Mr. and Mrs. Szmukler working for Établissements Reynier in the 1920s, identifiable on patents and in the technical press.

MOTS-CLÉS. ganterie, gants, inventeur, innovateur, innovation, Grenoble, Xavier Jouvin, Szmukler, industrie.

KEYWORDS. glove industry, gloves, inventor, innovator, innovation, Grenoble, Xavier Jouvin, Szmukler, industry.

«Sous le nom de ganterie, l'on entend l'art de fabriquer toute sorte de gants, espèce de vêtement de main destiné principalement à défendre du froid pendant l'hiver, et du hâle pendant l'été $[\ldots] »$ [DID, D'A 1751].

Porté hiver comme été, le gant a été au cours des siècles antérieurs un bien à usage quotidien, faisant partie intégrante de l'habillement [ROC 1991] comme en témoigne sa définition proposée par l'Encyclopédie de Diderot et $\mathrm{D}^{\prime}$ Alembert ${ }^{1}$. Parfumé, coloré, richement décoré, il est aussi un accessoire de mode «socialement indispensable » [COQ 2003] agrémentant la toilette féminine tout au long du XIX ${ }^{\mathrm{e}}$ siècle [COL, DAL 2022]. Jusqu'aux années 1950, « une femme des milieux aisés ne sortait pas sans chapeau et sans gants » [BOR 1988]. Ganterie de luxe pour la haute société [CoQ, BON 2015], [KAA, MON, SiM 2015], bon marché pour les plus modestes, les gants en tout genre sont produits dans des « microrégions dédiées au cuir » [LE B 2007] comme Paris [LAN 2015], Grenoble, Millau, Chaumont, Niort ou encore Saint-Junien. La ganterie s'inscrit dans la branche industrielle

\footnotetext{
${ }^{1}$ Je remercie Anne Dalmasso, Cédric PERRIN, Christophe CAPUANO, Florent Le Bot et Sophie Boutillier pour leurs précieux conseils dans l'élaboration de ce travail, ainsi que ainsi que Nicolas BroISIN, Perrine CAMUs, Camille CoRdIER, William FizE, Irène GIMENEZ, Raphaël LACHELLO, Elsa NEUVILLE et Éric SERGENT, doctorants au LARHRA, pour leurs suggestions et relectures.
} 
des cuirs et peaux qui a fait l'objet de riches études notamment autour des recherches conduites par Florent Le Bot [LE B 2005, 2007, 2008], [LE B, PER 2011] complétées par les travaux menés par Cédric Perrin sur les tanneries [PER 2015, 2014]. Elle intéresse également le champ de l'histoire environnementale, traitant des pollutions industrielles générées par ces industries [PER 2014], [BAR 2005]. Elle fait partie des activités marquées par une organisation complexe de la production, éloignée de celle de la grande entreprise, et par la persistance de formes variées d'organisation du travail tout au long du processus d'industrialisation [VER 1997].

À Grenoble, les gantiers s'établissent en corporation en 1702, date à laquelle un maître-gantier grenoblois agit pour la première fois au nom de celle-ci [ROU 1887]. La ville compte alors douze maîtres-gantiers [PRU 1888]. Témoignant de la consommation [ROC 1997], [DAU 2018] croissante de ce bien, leur nombre s'accroît progressivement pour atteindre 64 en 1787 [PRU 1888], entre 90 et 100 fabricants dans la décennie 1890 et 78 en 1920-1921 ${ }^{2}$. Jusque dans la seconde moitié du XIX ${ }^{\mathrm{e}}$ siècle, le gantier est un marchand-fabricant à la tête d'une affaire personnelle, inséré dans un réseau [FON 1984]. Le XIX ${ }^{\mathrm{e}}$ siècle voit naître de nombreuses sociétés de ganterie en nom collectif associant le fabricant de gants à des membres de sa famille ou à des mégissiers ou teinturiers dont dépend le gantier pour la préparation des peaux utilisées pour confectionner les gants. Dès les années 1870, si certaines ganteries conservent le modèle de l'atelier principalement appuyé sur un réservoir de main-d'œuvre à domicile [COL 2020a], d'autres rassemblent les travailleurs et travailleuses dans les locaux de l'entreprise, réalisent l'intégration d'une fabrique de cuir [LE B 2007] et tendent à adopter le modèle de l'usine sans toutefois aller au bout du processus de concentration puisque le travail à domicile persiste. Les entreprises composant cette partie industrialisée du secteur - au sens où des modifications sont repérées notamment dans les processus de fabrication et dans l'organisation du travail [LE B 2007] - recherchent en permanence le « progrès », la «nouveauté », la «modernité », tout un vocabulaire utilisé par les patrons en synonyme de ce que nous appelons aujourd'hui « innovation » [GAL, JUD, SAI 2004]. La thématique de l'innovation suscite un vif intérêt dans les années 1970 lorsque les travaux de Schumpeter [SCH 1974 et 1999] sont remis au goût du jour, intéressant différentes disciplines comme l'économie, le management, la sociologie, l'ingénierie [BOU, FOR, GAL, LAP, TAN 2014]. Dans le champ de l'histoire, l'innovation est pleinement introduite lorsque l'historien François Caron «pose les bases d'une véritable histoire de l'innovation » [GRI, FER 2007] en 1987 dans l'introduction d'un numéro de la revue Histoire, économie \& société [CAR 1987]. Dès lors, elle est placée au cœur de nombreuses études [BOU, GRI 2012]. Entendu ici au sens large comme un processus visant à introduire quelque chose de nouveau, un changement d'ordre scientifique, technologique, organisationnel ou encore commercial qui permet d'améliorer l'existant par la création et l'application de nouvelles connaissances [DRU 1993] dans un domaine donné, l'innovation s'appuie sur l'invention [JOB, TAL 2012] ou sur la recherche fondamentale ou expérimentale. Comme dans d'autres secteurs, en milieu gantier, «l'innovation est majoritairement le fait d'inventeurs indépendants » [LE B, MIC 2020] jusque dans la seconde moitié du XIX ${ }^{\mathrm{e}}$ siècle qui imaginent «un nouveau procédé ou un nouvel outil » pour « résoudre un problème technique donné » [MAT 2011]. Dès la fin du $\mathrm{XIX}^{\mathrm{e}}$ siècle, l'innovation dans la ganterie mobilise les ressources de la science et particulièrement la chimie. Elle s'appuie sur les compétences d'individus qualifiés, dont la ganterie n'est pas forcément le cœur de métier, recrutés à l'issue d'une formation dispensée dans des établissements nouvellement ouverts à l'image de l'École française de tannerie créée à Lyon en 1899 [PER 2014], [LE B en cours] ou encore de l'Institut électrotechnique de Grenoble en 1900 [ROB 2001] répondant aux besoins nouveaux ressentis par les industriels de la filière cuir, tandis que l'école de ganterie ouverte à Grenoble en 1910 met l'accent sur les compétences commerciales [COL 2021]. Ce sont généralement des chimistes et ingénieurs civils [JOL 2020] qui travaillent à

\footnotetext{
2 Pour les années 1890 et 1920, les chiffres sont issus d'un travail de recensement du nombre de ganteries dans les annuaires du département de l'Isère réalisé dans le cadre de ma thèse de doctorat en cours depuis 2017, portant sur l'histoire de la ganterie grenobloise de la Révolution à nos jours.
} 
l'amélioration des procédés et produits dans une entreprise dotée de «bureaux d'études » et/ou de «laboratoires » d'essais ${ }^{3}$.

Les acteurs de l'innovation du secteur gantier sont méconnus, restés dans l'ombre d'un individu nommé Xavier Jouvin (1801-1844) qui a fait l'objet de toute une littérature après sa mort le présentant comme le seul inventeur de la ganterie. Les biographies écrites en son honneur méritent d'être analysés pour montrer que le portrait dressé est une construction classique de la figure de l'inventeur héroïsé bien connue des historiens [DAL 2001], [MAC 2003], [JAR 2009]. Cet article s'attache à questionner la figure de Xavier Jouvin qui, érigé par l'élite locale au rang d'inventeur héroïque, devient un modèle au sein du milieu gantier et à montrer qu'il y a eu d'autres acteurs et actrices de l'innovation dans l'activité gantière restés dans l'ombre malgré des trajectoires passionnantes. Déconstruire la figure héroïsée de Jouvin permet d'inscrire les multiples inventions méconnues ayant contribué à l'amélioration des techniques de fabrication des gants, perceptibles dans les dépôts de brevets, dans processus d'invention collective largement étudié par l'historiographie [ALL 1983], [HIL, PER 2017], [HIL 2009]. Il s'agira donc dans un premier temps d'explorer la doxa héritée du XIX ${ }^{\mathrm{e}}$ siècle entourant le gantier Xavier Jouvin puis de montrer qui il était réellement et pourquoi il a été choisi comme l'inventeur représentatif de la ganterie. Dans un second temps, le rôle et la place des «travailleurs de l'innovation » [BOI, DID, YOU 2016] seront étudiés pour mettre au jour des parcours d'individus méconnus, à l'image du couple Szmukler travaillant pour la ganterie Reynier [COL 2020b] dans les années 1920. Si la première partie s'attache à analyser les écrits produits sur Xavier Jouvin au XIX ${ }^{\mathrm{e}}$ siècle, la deuxième s'appuie sur un corpus de sources peu exploré, voire inexploré auparavant concernant la ganterie, constitué entreautres du registre du commerce, d'actes de sociétés [JOL 1998, 2003], de plans d'usines, de la presse technique de l'industrie du gant et de notices produites par les gantiers à l'occasion des Expositions Universelles.

\section{Le temps de l'inventeur: Xavier Jouvin et la main-de-fer}

«L'invention de Xavier Jouvin, en 1832, qui a établi le Système des pointures, a été la seule découverte qui puisse être considérée comme ayant vraiment marqué une date dans les annales de l'industrie de la ganterie, tant au point de vue de la facilité de la vente. Le problème difficile d'adapter à chaque mesure de mains le gant ayant bien la dimension qui convient a été résolu par lui. On ne saurait donc trop rendre hommage à l'illustre inventeur, reconnaître la portée de son invention et l'influence qu'elle eut sur la réputation mondiale du gant de Grenoble. » [JAM 1925].

\subsection{La construction de la figure de l'inventeur dans l'activité gantière grenobloise}

$\mathrm{Au} \mathrm{XIX}^{\mathrm{e}}$ siècle, la figure de l'inventeur héroïque vient fédérer bon nombre de secteurs d'activité accompagnant ainsi la Révolution industrielle. Mythifié, l'inventeur érigé en héros permet aux gens du milieu de s'identifier à ce modèle construit par des auteurs du XIX ${ }^{\mathrm{e}}$ siècle, à l'image de la figure héroïsée de James Watt [MAC 2003], de l'inventeur du métier à tisser Jacquard permettant à François Jarrige de «questionner le processus de construction de la figure de l'inventeur en héros national » [JAR 2009] ou encore de l'analyse menée par Anne Dalmasso sur les récits construits autour d'Aristide Bergès [DAL 2001]. De la même manière, Xavier Jouvin s'est retrouvé hissé au premier rang de l'histoire du Dauphiné pour avoir inventé dans les années 1830 une méthode, un objet et des outils permettant de répondre à un problème rencontré dans la fabrication des gants. Ses inventions, qui portent son nom, visent à pallier le manque de régularité dans la coupe du gant, alors conçus dans l'à-peu-près pour les clients éloignés, ou sur mesure pour la clientèle de proximité, et à

\footnotetext{
${ }^{3}$ Sur la question des laboratoires d'essais, nous signalons l'HDR en cours de Florent Le Bot : « Innovation et recherche appliquée en cluster. Les laboratoires d'essais à l'articulation des écoles techniques supérieures, des entreprises, des territoires et de l'État, 1880-1960. » (garante Liliane Hilaire-Perez) dont l'École de tannerie de Lyon fait partie du terrain d'étude.
} 
fixer des pointures tenant compte des différents types de mains pour faciliter le choix du consommateur :

«L'ouvrier découpait lui-même les gants, c'est-à-dire qu'il faisait les doigts, le pouce et en déterminait l'emplacement. Aucun modèle n'existait; tout se faisait au petit bonheur et, si j'ose employer l'expression vulgaire, à vue de nez, si bien que deux paires de gants de même taille avaient rarement les mêmes dimensions » [BON 1919], selon les propos du Président d'honneur de la Chambre syndicale des fabricants de gants de Grenoble en 1919.

En réponse, Xavier Jouvin dépose un brevet en 1834 «pour des instruments destinés à la coupe des gants ${ }^{4}$ qui s'intègrent dans une méthode qu'il a élaboré consistant « à substituer à la coupe aux ciseaux, effectuée par l'ouvrier au jugé et suivant les données générales de l'expérience, une coupe mécanique à l'aide de calibres numérotés et établis méthodiquement $»^{5}$. Il dépose ensuite des brevets de perfectionnements et d'additions en 1835,1836 et $1838^{6}$. Le dernier correspond à un emporte-pièce mieux connu sous le nom de mains-de-fer qui permet de couper les gants mécaniquement par séries.

Ses hagiographes racontent que pour parvenir à ses découvertes, Jouvin mène une étude des différents types de mains à l'amphithéâtre de l'hôpital de Grenoble qui lui permet de repérer 322 types de mains, d'établir une série de trente-deux grandeurs et de dresser une classification de la main «qui, pour chaque grandeur, comprenait la forme : effilé, très effilé, moyen, large et très large ! » [BOU 1900]. La suite de ses expériences aboutit à la conception d'une « règle » sur laquelle sont inscrits « les trente-deux chiffres correspondant aux trente-deux élasticités de la peau réclamées par les types des grandeurs de la main » :

«Cette règle a donné naissance à une série de trente-deux modèles qui ont nom calibres. Le calibre est appliqué sur une peau et aussitôt la puissance d'élasticité de cette peau est déterminée d'une façon très approximative » [BOU 1900].

Après sa mort en $1844^{7}$, l'élite locale mobilise le schéma classique de l'inventeur hérö̈que en rédigeant une multitude de biographies élogieuses qui lui confèrent une grande notoriété à titre posthume. Suivant le mythe de l'inventeur-héros, ses hagiographes le décrivent comme un « rêveur » incompris de ses contemporains et qui s'est construit seul, faisant face à un parcours parsemé d'embûches. Du point de vue des hommes du XIX ${ }^{\mathrm{e}}$ siècle, l'essor de l'activité gantière est attribué à ses inventions qui auraient « révolutionné » cette industrie. Un véritable culte lui est voué dans la seconde partie du XIX ${ }^{\mathrm{e}}$ siècle. Son nom est donné à un quai de la ville de Grenoble en 1866, devenu une place sur laquelle une statue à son effigie est édifiée et inaugurée en 1889.

\footnotetext{
${ }^{4}$ Bulletin des lois du Royaume de France, janvier 1837, p. 183.

${ }^{5}$ Revue Ganterie, juin 1928, p. 419.

${ }^{6}$ Bibliothèque municipale (BM) Grenoble, Notice de la Maison Jouvin, 1871.

${ }^{7}$ Archives municipales (AM) Grenoble, registre de décès, année 1844, n ${ }^{\circ} 151$.
} 


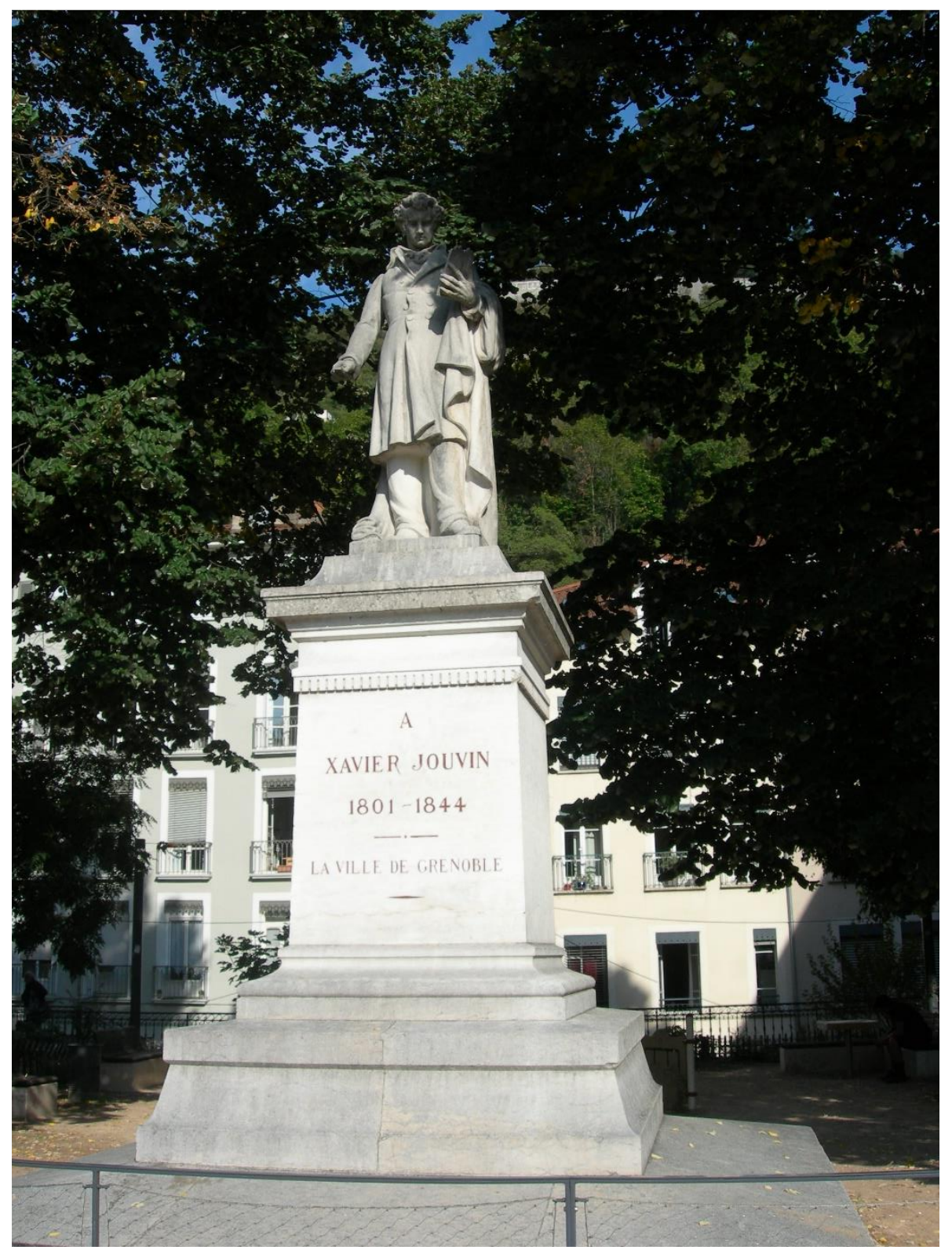

Figure 1. Photographie de la statue de Xavier Jouvin située à Grenoble sur la place Xavier Jouvin (source : auteur, 2020).

Pour élever cette statue, des souscriptions sont lancées à l'échelle locale au sein du milieu gantier. Un chant intitulé «Le chant national des gantiers hymne religieux et patriotique » est composé et vendu « au profit de la souscription au monument de Xavier Jouvin ${ }^{8}$. Les gantiers envisagent de le chanter lors des fêtes prévues pour l'inauguration de la statue. L'héroïsation de la figure de Jouvin transparait clairement dans les paroles :

«Le Christ, ô sagesse profonde,

N'était qu'un modeste ouvrier...

Ce fut de l'obscur atelier

Que sortit le vainqueur du monde... » ${ }^{9}$.

${ }^{8}$ BM Grenoble, V3004, pièce 16, Le chant national des gantiers.

${ }^{9}$ Ibid. 
Il est présenté comme le « régénérateur » de la ganterie et qualifié de « l'ami de l'humanité ». Le chant incite à suivre l'exemple de cet individu présenté en modèle : «Jurons tous [...], De poursuivre son noble exemple !».

\subsection{Le choix de Jouvin comme figure inventive de l'industrie du gant}

L'industrie du gant a trouvé son héros, son représentant, celui à qui s'identifier. Mais pourquoi lui ? Méconnu de son vivant, il est en réalité un inventeur parmi d'autres. Un bref coup d'œil dans les dépôts de brevets du XIX ${ }^{\mathrm{e}}$ siècle prouve que d'autres individus ont aussi tenté d'apporter du «progrès » dans la fabrication des gants. L'analyse des biographies dédiées à Xavier Jouvin montre que de son vivant, il plante les germes de sa notoriété future par des stratégies de valorisation visant à faire reconnaître de ses contemporains l'apport de ses inventions. Tout d'abord, il se présente à l'Exposition des produits de l'industrie française tenue à Paris en 1839 où ses talents d'inventeur sont salués pour la première fois lorsque le jury lui décerne une médaille de bronze, succès que ses hagiographes ne manquent pas de rappeler :

«Ce fabricant a apporté de la perfection dans l'ensemble des opérations ; il fait une grande quantité de gants, et ses produits sont estimés dans le commerce; il emploie beaucoup d'ouvriers. Le jury lui décerne une médaille de bronze. » [SCH 1839].

Ensuite, il a su se faire apprécier des ouvriers gantiers en devenant leur bienfaiteur : il apporte, à partir de 1839, une aide financière régulière équivalente à cinq centimes par douzaine de gants qu'il produit à la Société de secours mutuels des gantiers dont le but est de venir en aide aux ouvriers nécessiteux. Cette société masculine créée en 1803 à Grenoble par un ouvrier gantier nommé André Chevalier regroupe les principaux métiers de la ganterie [CAR 2017]. Ses effectifs sont importants, atteignant 1110 membres titulaires - «gantiers »- en $1872^{10}$. Suite à ce don, ses membres lui vouent un véritable culte en plaçant son portrait en têtes des livrets remis à chaque adhérent et son buste dans la salle de réunion après une cérémonie d'inauguration, selon ses hagiographes du XIX siècle. Remis en contexte, ce geste qui lui confère l'image d'un homme généreux et soucieux de la condition ouvrière est déterminant dans le processus de construction de sa notoriété dans la mesure où les effectifs ouvriers sont considérables dans la ganterie : 52 patrons emploient environ 470 ouvriers «du sexe masculin » ${ }^{11}$ en 1815 tandis qu'en 1860 une centaine d'établissements de ganterie emploie entre 1600 et 1650 ouvriers $^{12}$, non compris le nombre plus important encore d'ouvrières.

Enfin, l'ascension sociale de ce grenoblois fait de lui un modèle de réussite pour le milieu ouvrier. Il débute comme apprenti, puis devient ouvrier avant d'intégrer le patronat lorsqu'il prend la tête de la ganterie familiale dans les années 1830 avec son demi-frère. Sa famille s'inscrit définitivement dans la bourgeoisie industrielle locale lorsque sa fille Rose-Odile-Marie Jouvin (1843-1918) - issue de son mariage avec Julie-Thérèse Rey (1819-1891) ${ }^{13}$ - épouse Edouard Rey en 1866 qui devient maire de Grenoble entre 1881 et 1888.

\subsection{Du mythe à la réalité : Xavier Jouvin, l'archétype du gantier grenoblois}

Plus intéressante que la figure classique de l'inventeur-héros construite au XIX ${ }^{\mathrm{e}}$ siècle, la réalité montre qu'il est l'archétype du gantier. Grenoblois d'origine, Xavier Jouvin est issu d'un milieu de

${ }^{10}$ AM Grenoble, 5Q44, Société de Bienfaisance et de prévoyance mutuelle des gantiers de Grenoble, Compte-rendu des recettes et des dépenses, 1871-1872.

${ }^{11}$ Archives départementales (AD) 38, 138M1, «n³ Liste des 60 manufacturiers ou fabricants $-1815 »$.

12

$\mathrm{AD} 38,138 \mathrm{M} 10$, Situation industrielle du département de l'Isère année 1860.

13

AD 38, fonds Jouvin, 26J103, Arbre généalogique de la famille Jouvin. 
petits négociants dauphinois ancrée dans la ganterie depuis le XVIII ${ }^{\mathrm{e}}$ siècle. Il naît en $1801^{14}$ d'un père successivement «peigneur de chanvre », «liquoriste » puis «marchand gantier ${ }^{15}$. Comme la plupart des ganteries de ce temps, la maison Jouvin s'appuie sur des réseaux familiaux dans lesquels Xavier s'insère progressivement, d'abord comme apprenti dans l'entreprise familiale à l'âge de douze ans environ, après avoir reçu une instruction primaire, puis en tant qu'ouvrier dans la première partie de sa vie d'adulte. En 1817, son père ouvre un magasin de gant à Paris, rue SaintDenis, pour vendre les gants fabriqués par son oncle dans l'atelier grenoblois ${ }^{16}$. Xavier Jouvin reprend l'affaire familiale dans les années 1830 avec son demi-frère.

Loin de l'image de l'inventeur en marge qui se construit seul, son parcours de vie est typiquement celui d'un fils de gantier du XIX ${ }^{\mathrm{e}}$ siècle qui, pour la génération antérieure, est perçu comme un futur héritier à former en vue d'une succession à venir. Au sein d'une société de ganterie, les fils acquièrent le statut d'associés à partir du moment où ils sont jugés aptes à reprendre l'affaire. Ainsi, il est normal que malgré son implication, Xavier Jouvin ne soit pas toujours inclus dans les différentes sociétés successives créées entre les membres de la famille entre 1820 et 1840 et qu'il travaille dans la ganterie familiale bien avant l'officialisation de son intégration par le statut d'associé. Il ne s'agit donc pas d'un manque de confiance lié à ses activités inventives mal vues par sa famille comme ont pu l'écrire ses hagiographes suivant le schéma bien connu de l'inventeurhéros.

\section{L'ère de l'innovation: la mobilisation de la science dans la ganterie}

Si les inventeurs et inventrices de la ganterie méconnus au XIX ${ }^{\mathrm{e}}$ siècle sont masqués par la figure de Jouvin, celle-ci continue de faire de l'ombre aux acteurs de l'innovation du $\mathrm{XX}^{\mathrm{e}}$ siècle qui sont également cachés derrière la renommée des entreprises pour lesquelles ils travaillent. À partir des années 1870, ces individus, dont les compétences sont reconnues, contribuent activement à la réussite des entreprises composant la partie industrialisée du secteur gantier, objet de notre propos. Tandis que des entreprises appartenant à d'autres secteurs d'activité se dotent de laboratoires d'essais ou de recherches dès le début du XIX ${ }^{\mathrm{e}}$ siècle comme l'a montré Michel Cotte dans son étude consacrée aux entreprises de Marc Seguin et ses frères [CОТ 2007], ce type de structure fait son apparition dans la ganterie dans le dernier tiers du $\mathrm{XIX}^{\mathrm{e}}$ siècle. C'est à cette période qu'émerge la recherche en entreprise ${ }^{17}$ dans le secteur gantier repérable par les «laboratoires » d'essais bien que, comme dans d'autres secteurs, ils ne soient pas la seule forme d'organisation en la matière [GAL 2008], des associations de compétences ayant également été repérées dans les archives.

\subsection{La naissance de la recherche en entreprise}

« [...] Il restait, toutefois, beaucoup à faire du côté de la fabrication, spécialement dans la mégisserie et la teinture des peaux, et les efforts de nos industriels se sont dirigés dans cette voie avec des résultats souvent heureux. Certains produits employés ont depuis longtemps disparu; des machines exécutent maintenant les travaux pénibles, et souvent malsains, que faisaient jadis les ouvriers. » [JAM 1925].

14 AM Grenoble, registre de naissance, année $1802, \mathrm{n}^{\circ} 212$.

15 AD 38, fonds Jouvin, 26J103, Arbre généalogique de la famille Jouvin.

${ }^{16}$ AD 38, fonds Jouvin, 26J.

17 Sur ce sujet voir notamment : LE Roux M., L'entreprise et la recherche : un siècle de recherche industrielle à Pechiney, 1808 1996, Rive Droite, Paris, 1998 ; CHEZEAUX N., De la forge au laboratoire. Naissance de la métallurgie physique (1860-1914), 
Ces quelques lignes écrites par le Président de la Chambre syndicale des fabricants de gants de Grenoble en 1925 expriment bien cette quête de ce que les gantiers appellent le «progrès » technique dans laquelle entre le secteur dans le dernier tiers du XIX ${ }^{\mathrm{e}}$ siècle. Elles évoquent également les acquis de l'industrialisation partielle de certaines entreprises de ganterie [COL 2020a] qui, dès les années 1870 environ, réalisent l'intégration des activités de mégisserie et/ou de teinturerie [LE B 2007] en amont, passent de l'atelier à l'usine et mettent en place une organisation productive nouvelle. Les entreprises cherchent alors à réaliser des économies d'échelle, à standardiser la production tout en améliorant la qualité des produits et proposent des nouveautés pour satisfaire les exigences du consommateur. L'innovation est omniprésente dans leurs processus de production et de commercialisation des gants. Ainsi, de nouveaux services sont ouverts dans les entreprises au gré des besoins : «laboratoires de recherche et d'essais », «bureaux d'études", «services de création», bureaux de promotion, etc. voient le jour mobilisant un nouveau type de personnel. L'innovation est donc « le produit d'un travail collectif» [LE B 2020] qui repose sur la collaboration des acteurs composant les différents services de l'entreprise et sur les liens entre les acteurs du milieu gantier et plus largement de la filière cuir. Les «travailleurs de l'innovation » [BOI, DID, YOU 2016] n'ont pas la ganterie pour cœur de métier : ce sont des chimistes, des ingénieurs, des techniciens, des dessinateurs, des modélistes, etc. Si les sources disponibles ne permettent pas de présenter le fonctionnement concret des structures internes aux entreprises dans lesquelles ils travaillent, il est toutefois possible de cerner le rôle du «laboratoire » et du «bureau d'étude » dans le processus d'innovation à travers l'étude conjointe de la presse technique, de documents produits à l'occasion des Expositions Universelles et de plans d'usines pour les ganteries Perrin et Reynier.

Les «laboratoires » et «bureaux d'études » interviennent dans la phase de préparation des peaux qui passent par la mégisserie puis la teinturerie avant la conception du gant en lui-même. Dans ces domaines, la ganterie a largement bénéficié de l'avancée des techniques et notamment du passage du tannage végétal au tannage au chrome qui s'opère à l'entrée dans le $X^{\mathrm{e}}$ siècle [PER 2014]. Ces «laboratoires » ont pour mission l'amélioration des techniques pour permettre gain de temps et économie d'échelle ainsi que la recherche de procédés innovants suivie de leur mise en application. La naissance de ces structures est permise grâce à l'avancée majeure de la chimie industrielle [LAM, STO 2010] et au développement de la formation répondant aux besoins nouveaux ressentis par les industriels de la filière cuir. Au début du $\mathrm{XX}^{\mathrm{e}}$ siècle, l'emploi d'ingénieurs et de chimistes se généralise dans les entreprises de ganterie «modernes", selon le terme utilisé par les gantiers contemporains de cette période. Ainsi, en 1919, lorsque l'entreprise Perrin cherche à recruter un nouveau chimiste capable de travailler à la fois la teinture du cuir et du tissu pour gants pour son usine grenobloise, les dirigeants s'adressent à l'école de tannerie lyonnaise. Toutefois, comme le souligne l'historienne Anne Dalmasso dans ses travaux portant sur l'entreprise Neyrpic-Sogreah, il convient de «relativiser le terme de "laboratoire" » [DAL, ROB 2007] car il s'agit, dans le cas de la ganterie, de modestes installations au sein des usines et la documentation disponible ne fournit pas d'informations quant au coût de ces structures.

\subsection{Le cas de la ganterie Perrin à la fin du XIXe siècle}

À Grenoble, dans le secteur de la ganterie, le premier «laboratoire » repéré dans les sources est celui de l'entreprise Perrin. Une petite structure de recherche est installée dans ses locaux lorsqu'elle se dote de sa propre teinturerie en 1887 équipée d'un «laboratoire » conçu comme un «atelier spécial où sont employés 1 chimiste maître teinturier, 4 employés ou contremaîtres, 25 ouvriers teinturiers et 18 ouvriers palissonneurs $»^{18}$ en 1894 . Ce « laboratoire » a pour mission d'améliorer la teinture des peaux en couleurs, d'obtenir de nouvelles nuances et, de manière générale, d'améliorer les procédés de teinture. À l'occasion des Expositions Universelles, la Maison Perrin vante les résultats obtenus en son sein qu'elle juge innovant. Parmi eux, l'un est particulièrement remarqué de

18 BM Grenoble, Notice sur la maison Perrin frères et Cie, Manufacture de gants à Grenoble, 1894. 
ses contemporains : l'urine humaine, utilisée comme mordant, est remplacée par un composé chimique suite aux découvertes de Jules Mornet, chimiste de la ganterie Perrin et « inventeur » de machines pour la préparation des peaux, dont la documentation disponible ne permet pas de retracer le parcours. Cette découverte majeure à l'échelle locale à l'heure où les innovations demeurent le secret de l'entreprise est approuvée par le Conseil d'Hygiène de Grenoble en 1897 :

« [...] après avoir pris connaissance du mémoire de M. Mornet [...] j'ai pensé qu'il serait bon de visiter les ateliers de teinture de MM. Perrin Frères \& $C^{\text {ie }}$ situés à l'IleVerte, à Grenoble.

Le 2 juillet, je m’y suis présenté inopinément. J'ai constaté que le mordant artificiel en question y avait complètement remplacé l'urine depuis plus de deux ans. Je n'ai trouvé d'urine nulle part, et plusieurs ouvriers, consultés isolément, m'ont affirmé qu'il n'y en avait point.

Dans cette teinturerie, le mélange qui remplace l'urine se fabrique sous un abri spécial, dans des fûts à fermeture hydraulique et il n'en résulte aucune odeur.

Dans l'atelier de teinture où on l'emploie on sent une légère odeur ammoniacale nullement répugnante. [...]

La recherche d'un mordant capable de remplacer l'urine putréfiée n'est pas nouvelle. Diverses préparations ont depuis longtemps été essayées dans ce but, mais aucune d'elles n'avait donné jusqu'ici de bons résultats et, après en avoir essayé, les fabricants en étaient toujours revenus à l'urine.

Le mordant artificiel de M. Mornet a seul, jusqu'à présent, résisté victorieusement à une expérience de plusieurs années faite sur une grande échelle. Son emploi rend désormais inutile celui de l'urine putréfiée, matière répugnante et éminemment suspecte, et il constitue, à notre avis, un progrès considérable au point de vue de l'hygiène. »19.

Si la ganterie grenobloise constitue notre terrain d'étude, ce type de structure ne concerne pas uniquement les ganteries de Grenoble. Les recherches menées par Florent Le Bot ont montré qu'en septembre 1944, la SA Tréfousse de Chaumont «emploie encore six ingénieurs-chimistes et neuf techniciens et dispose d'un laboratoire pour le développement des méthodes de tannage qui demandent une très bonne maîtrise de la chimie » [LE B 2007].

19 BM Grenoble, Rapport sur le mordant remplaçant l'urine putréfiée dans les teintureries pour peaux de gants établi par le Conseil d'hygiène de Grenoble, 29 juillet 1897. 


\subsection{L'arrivée du chimiste et de l'ingénieur dans les entreprises de ganterie : l'exemple du couple Szmukler}

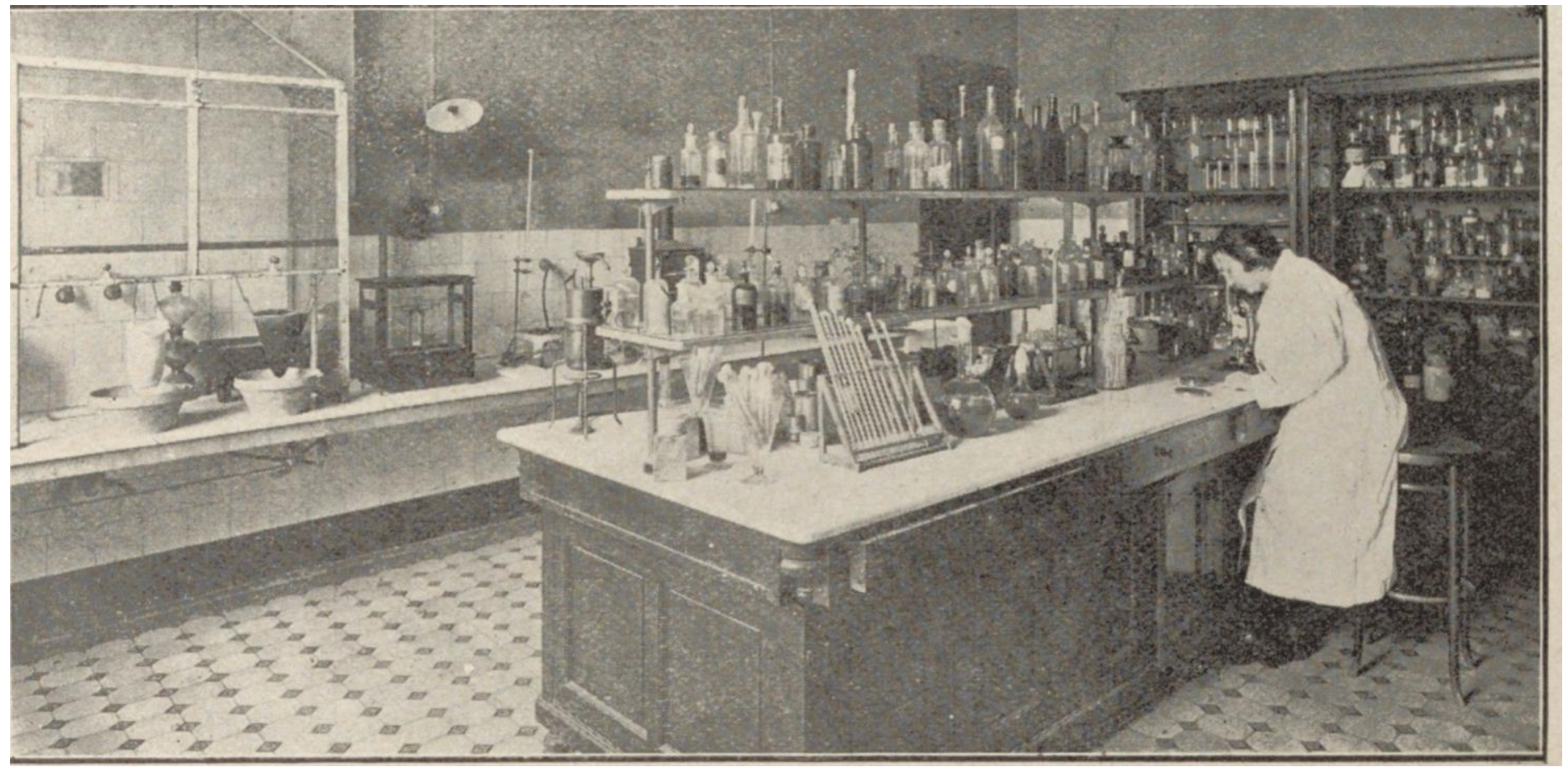

Figure 2. Madame Szmukler au travail dans le « laboratoire » de l'usine de mégisserie Reynier (Source : gallica.bnf.fr / BnF, Revue Ganterie, juillet 1926, p. 446)

D'un profil quelque-peu similaire à l'ingénieur-chimiste de chez Perrin, la chimiste et l'ingénieur employés par la ganterie Reynier ont laissé quelques traces dans les archives permettant de retracer dans les grandes lignes leur trajectoire captivante, sans toutefois pouvoir approfondir faute de documents. Créée en 1832, la Maison Reynier investit dans des structures de recherches dans la décennie 1910 après avoir réalisé son intégration verticale à la fin du XIX ${ }^{\mathrm{e}}$ siècle. Le « laboratoire » de chimie et la pièce attenante, nommée dans les sources «mégisserie et teinturerie d'essai », sont ouverts au cœur de l'usine de mégisserie édifiée en 1913, comme le confirment les plans du bâtiments ${ }^{20}$, permettant un travail conjoint entre la directrice du laboratoire et les ouvriers et ouvrières. La direction du « laboratoire » est confiée à $\mathrm{M}^{\mathrm{me}}$ Szmukler, chimiste de métier, ce qui ne manque pas de surprendre ses contemporains comme le montrent ces quelques lignes publiées en 1922 dans un journal local de la région dauphinoise :

«Quand M. Gaston Vidal, sous-secrétaire d'État à l'Enseignement technique, vint à Grenoble [...] pour poser la première pierre de la nouvelle école Vaucanson, il eut l'occasion de visiter la fabrique de gants Reynier, l'une des plus importantes de la cité de la ganterie. Il fut surpris de trouver à la tête du laboratoire de chimie, - que comporte toute ganterie réputée - une femme, Mme Szmukler, et d'apprendre que cette femme venait de découvrir un procédé appelé à révolutionner la tannerie » ${ }^{21}$.

Si $\mathrm{M}^{\text {me }}$ Szmukler est la seule femme repérée travaillant à la recherche dans les entreprises de ganterie, dans d'autres secteurs, la place des femmes dans le milieu de l'invention et de l'innovation a été démontrée par l'historiographie dès les années 1990 [CHA 2009], [KIR, SMI 2004], [STA 1993]. $\mathbf{M}^{\mathrm{me}}$ Szmukler met ses compétences au service des Établissements Reynier vers 1918 jusqu'au début des années 1930. Son travail consiste à réduire le temps de transformation des peaux

20 AM Grenoble, 7Fi2874, plan de la mégisserie Reynier.

21 Journal Le Petit Dauphinois, le grand quotidien des Alpes Française, 5 décembre 1922. 
pour gants, à chercher des procédés novateurs pour produire de meilleures qualités de peaux et de nouvelles nuances de teintures, à fabriquer les produits chimiques utilisés dans l'usine, ou encore à faire des essais en vue de concevoir de nouveaux produits comme le gant lavable qui nécessite une recherche en amont pour rendre la peau teinte lavable à l'eau et au savon :

« Je recherche des cultures bactériologiques qui donnent les meilleurs résultats, je fais des expériences dans la mégisserie miniature attenant au laboratoire ; une fois que je suis arrivée au résultat, j'envoie les directions à l'ouvrier qui n'a qu'à les suivre. Je puis donc exercer un contrôle parfait et être sûre d'avance des résultats. ${ }^{22}$, explique Mme Szmukler à des étudiants américains en séjour à Grenoble venus visiter les usines Reynier.

Cette femme au parcours exceptionnel est originaire d'une famille dauphinoise. Désignée ainsi jusqu'à son décès en 1977, Szmukler est son nom d'épouse. Elle s'appelle Pauline-Léonie née sous le nom de Marcellot en 1887 à la Tour-du-Pin en Isère, fille d'Achille-Pierre Marcellot exerçant la profession de «directeur de l'École laïque » et de son épouse Julie Léonie Montmayeur, «sans profession ${ }^{23}$ selon l'état civil. Elle a étudié à la Faculté des Sciences de Grenoble où elle est «licenciée ès-sciences ${ }^{24}$ puis devient préparatrice des travaux du professeur de chimie Albert Recoura (1862-1945) dans cette même Faculté. Elle occupe ensuite une fonction similaire à l'Institut électrotechnique de Grenoble, où elle rencontre Constantin Szmukler alors étudiant dans cette école, qu'elle épouse en 1921.

Dans les années 1920, le couple vit à proximité de l'usine de mégisserie Reynier, dans un logement de fonction mis à disposition par l'entreprise ${ }^{25}$. De leur union naît un enfant unique en 1928 : une petite fille prénommée Huguette, aujourd'hui (en 2021) âgée de 93 ans, devenue $\mathrm{M}^{\mathrm{me}}$ Poncin-Szmukler, que nous avons eu le plaisir de rencontrer durant la période d'écriture de cet article. Pauline-Léonie Szmukler laisse le souvenir d'une femme discrète «qui n'aime pas qu'on parle d'elle ${ }^{26}$ mais aux qualités intellectuelles remarquables. Il est certain qu'à l'échelle locale et dans le milieu dans lequel elle évolue, ses compétences sont reconnues. En 1922, un journal local publie un article élogieux au sujet de son travail sous le titre : «Une femme a découvert un nouveau procédé pour mégir et teindre les peaux ${ }^{27}$. L'article met en avant la réduction des coûts de production obtenue grâce à l'une de ses découvertes et retranscrit les propos qu'elle tient au sujet de son travail :

«[...] les peaux d'agneaux ou de chevreaux, comme celles de toutes les autres bêtes, d'ailleurs, continuent à avoir une vie organique. C'est la bactériologie appliquée à l'industrie du gant qui constitue toute ma découverte. J'ai appliqué mes connaissances médicales à l'examen des peaux qui doivent servir à fabriquer les gants ; j'ai analysé ces peaux. J'ai étudié les phénomènes qui se produisent dans leur teinture, au cours de laquelle les éléments bon ou mauvais de l'organisme se contrarient.

\footnotetext{
${ }^{22}$ Revue Ganterie, juin 1924.

23 Archives de la famille Poncin, Acte de naissance de Pauline-Léonie Marcellot.

${ }^{24}$ Revue Ganterie, juillet 1926, p. 446.

25 Entretien avec Huguette Poncin-Szmukler.

${ }^{26}$ Selon les propos de Huguette Poncin-Szmukler.

27 Journal Le Petit Dauphinois, le grand quotidien des Alpes Française, 5 décembre 1922.
} 
C'est ainsi que j'en suis arrivée à étudier l'influence des maladies dont étaient atteintes les bêtes, sur la valeur de leur peau respective. Et je puis dire qu'au microscope cette influence est considérable. Il est même surprenant que l'on ne s'en soit pas préoccupé jusqu'ici. [...]

Vous dire quelle est exactement la méthode employée pour mégir, puis teindre les peaux, je ne puis le faire encore. Expérimentée depuis un an, elle donne les résultats les meilleurs, mais le secret qu'elle comporte est un peu la propriété de la ganterie Reynier qui a mis à ma disposition un laboratoire de premier ordre, et c'est elle qui décidera de l'heure où ma découverte pourra être divulguée.

Mais je puis vous affirmer que nous obtenons, dès maintenant, une économie énorme sur le prix de revient... ${ }^{28}$.

En complément des recherches menées dans le «laboratoire de chimie », l'entreprise dispose dans les années 1920 d'un «Bureau d'Études » ${ }^{29}$ dans lequel des ingénieurs et techniciens travaillent au perfectionnement des machines existantes et à la conception de nouvelles pour réaliser des économies d'échelle et régulariser la production au stade de la préparation des peaux. Le bureau d'études est placé sous la direction de l'ingénieur Constantin Szmukler qui intègre l'entreprise Reynier quelques années après sa femme en qualité d'ingénieur avant d'endosser la fonction de «directeur de la mégisserie et tannerie des Établissements Reynier ${ }^{30}$. La trajectoire de cet homme est tout aussi passionnante que celle de son épouse. Naturalisé Français en $1926^{31}$, KoifmanConstantin Szmukler, de son nom complet, est né en Pologne le 4 octobre $1891^{32}$. Les sources disponibles ne permettent pas de dater avec précision son arrivée en France ni de connaître les conditions dans lesquelles elle s'effectue. Selon sa descendance, il aurait fait la première partie de ses études en langue russe sur le sol polonais ${ }^{33}$. Il arrive à Grenoble en 1910, à l'âge de 19 ans ${ }^{34}$, où il achève son parcours universitaire avec l'obtention d'un «diplôme d'Ingénieur-Électricien » en $1915^{35}$ à l'Institut électrotechnique. Selon des interviews qu'il accorde à la presse locale en 1990 à l'approche de ses cent ans, il fait le choix de l'émigration vers la France pour une vie meilleure : " "Venant de Russie, où mon père dirigeait une fabrique de tissus, la France représentait pour moi la terre de tous les espoirs, de toutes les chances." $\gg{ }^{36}$.

Constantin Szmukler est l'inventeur de plusieurs machines à usage de la mégisserie et de la teinture pour gants brevetées en son nom accolé à celui des Établissements Reynier. Il fait partie des rares inventeurs du milieu gantier à avoir pu faire inscrire son nom sur les brevets déposés pour les inventions dont il est l'auteur. À l'inverse des inventeurs du XIX ${ }^{\mathrm{e}}$ siècle tel Jouvin, Constantin

28 Ibid.

${ }^{29}$ Revue Ganterie, novembre 1926.

${ }^{30}$ Ibid., septembre 1931, p. 761.

31 Journal officiel de la République française. Lois et décrets, 01-02-1926, p. 1523.

32 Ibid.

33 Entretien avec Huguette Poncin-Szmukler.

34 Journal Les affiches de Grenoble et du Dauphiné, 12 octobre 1990, article « Le doyen des chefs d'entreprise français est Grenoblois », p. 3.

35 Archives de la famille Poncin, Diplôme de Constantin Szmukler, 1915.

36 Journal Les affiches de Grenoble et du Dauphiné, op. cit. 
Szmukler est ingénieur de métier, il perçoit une rémunération pour inventer dans le cadre de l'entreprise et il est reconnu par ses pairs. Au début des années 1930, il se voit attribuer un prix de 25000 francs pour son «mémoire sur l'emploi de l'aluminium dans la mégisserie et la tannerie » dans le cadre du Concours International de l'Aluminium ouvert « aux techniciens du monde entier et destiné à récompenser les meilleures suggestions qui seraient faites au sujet des emplois de l'aluminium et de ses alliages $» .^{37}$ Ses recherches et inventions s'inscrivent dans la continuité de l'évolution des techniques employées dans la tannerie comme le passage du tannage végétal au tannage minéral qui constitue un gain de temps considérable dans le travail des peaux [PER 2014].

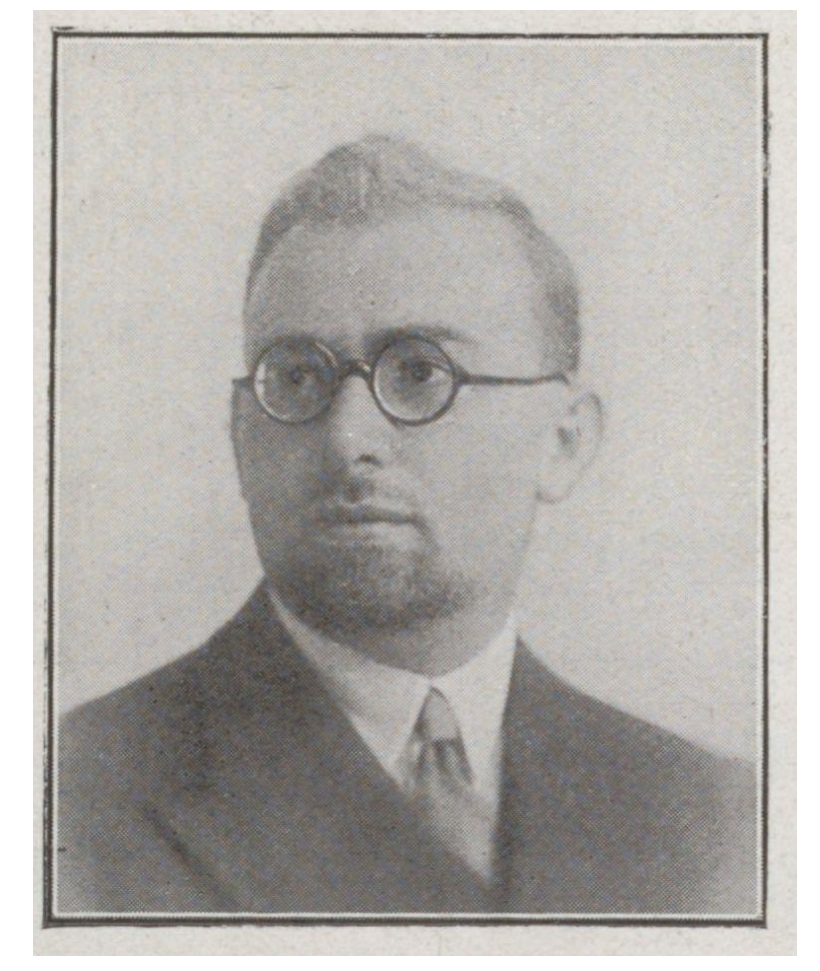

Figure 3. Portrait de Constantin Szmukler vers 1930 (Source : gallica.bnf.fr / BnF, Revue Ganterie, septembre 1931, p. 761).

Sans connaître la date exacte de l'entrée de Constantin Szmukler au service des Reynier, sa fille Huguette Poncin-Szmukler se souvient qu'il a travaillé à Lyon dans un autre secteur d'activité avant d'intégrer l'industrie du gant. La ganterie ne fut qu'épisodique dans le parcours de vie de ce couple «d'intellectuels» passionné par le travail, selon les souvenirs de leur fille, mais marque le départ d'une véritable collaboration professionnelle. Constantin Szmukler quitte les Établissements Reynier vers 1932 [PON 2012] pour lancer ses propres projets auxquelles il se consacrera jusqu'à sa mort en 1991. En 1934, il ouvre sa propre entreprise «Renodex » à Grenoble, une société qui s'occupe des « doigtiers, œillets et cathodes tubulaires pour la Radiotechnique » [PON 2012]. ${ }^{\text {me }}$ Szmukler suit son mari dans cette nouvelle aventure et laisse de côté sa carrière de chimiste dans les années 1930.

\section{Conclusion}

Étudier inventions et innovations dans l'activité gantière sur un siècle, des années 1830 aux années 1930, permet de mettre en lumière le passage d'un gant sans taille conçu dans « l'à-peuprès » au début du XIX ${ }^{\mathrm{e}}$ siècle à un gant design et bien taillé faisant l'objet d'une recherche à tous les stades de production au début du $\mathrm{XX}^{\mathrm{e}}$ siècle afin de créer la demande lorsque le gant de peau est de plus en plus délaissé.

37 Journal La Machine moderne : journal mensuel, octobre 1931, p. 753. 
Les progrès techniques apparus dans l'industrie du gant au XIX $\mathrm{e}^{\mathrm{e}}$ siècle et durant les trois premières décennies du siècle suivant sont permis grâce aux inventions et innovations qui se sont succédés sur près de deux siècles. Celles-ci sont le fait d'hommes et de femmes souvent méconnus à l'exception du gantier Xavier Jouvin érigé au rang de héros local à titre posthume, masquant ainsi les autres acteurs de l'innovation. Son image glorifiée reste d'ailleurs à l'échelle locale l'un des rares souvenirs de la ganterie grenobloise conservé dans la mémoire collective. La statue à son effigie élevée sur une place publique de la ville de Grenoble est encore debout et une partie de la maison dans laquelle il a vécu, et qui abritait autrefois la ganterie Jouvin fermée en 1964, a été transformée en Musée privé en 2012 par l'un de ses descendants.

Si l'apport de Xavier Jouvin à l'industrie du gant est certain, il marque le point de départ d'une longue quête de l'innovation dans l'activité gantière. Ce gantier qui s'est improvisé inventeur à ses heures perdues a été une source d'inspiration pour plusieurs générations de gantiers grenoblois qui, après lui, ont tenté d'établir leurs propres méthodes et inventions pour faire progresser la fabrication des gants ou de perfectionner son œuvre. Il est aussi devenu une source d'inspiration pour les érudits $\mathrm{du} \mathrm{XIX}^{\mathrm{e}}$ siècle qui posent les bases du mythe de l'inventeur autour de sa personne. La construction de ce mythe de l'inventeur héroïque de la ganterie grenobloise a permis de fédérer les acteurs de la ganterie autour d'un individu qui répond à différents critères, faisant de lui une figure représentative du gantier grenoblois de son temps et un modèle de réussite.

À partir des années 1870, l'ouverture de «laboratoires » et de «bureaux d'études » au sein de certaines entreprises de ganterie favorise le processus d'innovation. Les individus employés dans ces structures ont un profil différent de l'inventeur de la période précédente. Ce sont des chimistes, des ingénieurs ou des techniciens qui ont reçu une formation solide et perçoivent un salaire pour leur travail inventif. Cependant, si ces professionnels employés à la recherche en entreprise du début $\mathrm{du} \mathrm{XX}^{\mathrm{e}}$ siècle sont reconnus par leurs pairs, comme le montrent les distinctions honorifiques que certains ont pu recevoir, ils évoluent non seulement dans l'ombre de l'entreprise mais aussi dans celle de la figure héroïque de Xavier Jouvin que les acteurs de la ganterie admirent jusqu'à l'arrêt quasi-total de l'activité gantière dans les années 1960-1970. Pourtant, le XX $X^{\mathrm{e}}$ siècle a aussi eu son lot de figures exceptionnelles contribuant à la progression des techniques de fabrication des gants, à l'image des époux Szmukler. S'ils ne sont pas restés longtemps au service de l'entreprise Reynier, la ganterie fut pour eu un moyen d'ascension professionnelle. Les découvertes de Constantin Szmukler, lorsqu'il travaillait pour la ganterie Reynier, lui ont permis d'ouvrir sa propre entreprise pour exploiter ses procédés dans les années 1930. Cette entreprise dans laquelle il a concentré son énergie jusqu'à sa mort est encore en activité aujourd'hui, dirigée par sa descendance, et fait partie intégrante du tissu industriel du bassin grenoblois qui a connu de profondes mutations durant la seconde moitié du $\mathrm{XX}^{\mathrm{e}}$ siècle [DAL 2009], période à laquelle la ganterie s'éteint peu à peu.

\section{Sources imprimées}

[BON 1919] BONDAT E., «L'industrie gantière » dans Le Dauphiné au travail, Grands Établissements de l'Imprimerie Générale, Grenoble, 1919, p. 226.

[Bou 1900] Bouillat A., Les célébrités contemporaines du Dauphiné, Maison de la Bonne Presse, Grenoble, 1900.

[DID, D'A 1751] DIDEROT et D'AlEMBERT, Encyclopédie ou dictionnaire raisonné des sciences, des arts et des métiers, tome 17, 1751-1765.

[JAM 1925] JAMMET H., «L'industrie gantière », dans Grenoble et sa région 1900-1925, Allier père et fils, Grenoble, 1925.

[Pru 1888] Prudhomme A., Histoire de Grenoble, A. Gratier Éditions, Grenoble, 1888, p. 563.

[Rou 1887] RouX X., La corporation des gantiers de Grenoble avant et après la Révolution, Éditions Gabriel Dupont, Grenoble, 1887, p. 7. 
[SCH 1839] SCHLUMBERGER C. (Rapporteur), dans Catalogue officiel des produits de l'industrie française admis à l'exposition publique dans le carré des fêtes aux Champs-Elysées : année 1839 (2 édition), $8^{\mathrm{e}}$ commission, arts divers, 1839 , p. 431.

\section{Bibliographie}

[All 1983] Allen R. C.., «Collective Invention», Journal of Economic Behavior and Organization, n4, p. 1-24, 1983.

[BAR 2005] BARET-BOUGOIN E., La ville industrielle et ses poisons : les mutations des sensibilités aux nuisances et pollutions industrielles à Grenoble, 1810-1914, Presses Universitaires de Grenoble, 2005.

[BoI, Did, You 2016] BoISARD P., DIDRY C., Younes D. (dir.), Les travailleurs de l'innovation. De l'entrepreneur aux salariés, Presses Universitaires Rennes, Rennes, 2016.

[BOR 1988] Borne D., Histoire de la société française depuis 1945, Paris, Éditions Armand Colin, 1988.

[Bou, For, Gal, Lap, Tan 2014] Boutillier S., Forest J., Gallaud D., Laperche B., Tanguy C., et al.. Principes d'économie de l'innovation, Peter Lang, 2014.

[BOU, Gri 2012] Griset P. et BouviER Y., « De l'histoire des techniques à l'histoire de l'innovation. Tendances de la recherche française en histoire contemporaine », Histoire, économie \& société, n², p. 29-43, 2012.

[CAR 2017] CARANTON J., Les fabriques de la "paix sociale". Acteurs et enjeux de la régulation sociale (Grenoble, 1842-1938), Thèse de doctorat, Université Grenoble-Alpes, 2017.

[CAR 1987] CARON F., «L'innovation et l'histoire », Histoire, économie et société, n², p. 149-154, 1987.

[ChA 2009], ChanteuX A., «Les inventives. Femmes, inventions et brevets en France à la fin du XIX siècle », Documents pour l'histoire des techniques, n¹7, p. 90-97, 2009.

[Col, Dal 2022] Colonel A., Dalmasso A., Fait main : Quand Grenoble gantait le monde, Glénat, Grenoble, à paraître en 2022.

[Col 2021] Colonel A., «Histoire de l'"école" de ganterie de Grenoble (vers 1890-vers1920) : Des tentatives de mise en école de la formation masculine au métier de gantier », La Pierre et l'Écrit. Revue d'histoire et du patrimoine en Dauphiné, 2021, à paraître.

[COL 2020a] COLONEL A., «La ganterie grenobloise des années 1830 aux années 1930 : entre artisanat et industrie », dans Laloux L., Palaude S., Peters A. (dir.), Textile et habillement, Métiers d'autrefois, tome 1, Presses Universitaires de Valenciennes, 2020.

[COL 2020b] Colonel A., «La ganterie "moderne" a-t-elle existé ? L'exemple de la Maison Reynier, du "maîtregantier" à la Société Anonyme (1832-années 1930) », La Pierre et l'Écrit. Revue d'histoire et du patrimoine en Dauphiné, n³0, p. 141-165, 2020.

[CoQ, Bon 2015] COQuery N., Bonnet A. (dir.), Le commerce du luxe. Le luxe du commerce. Production, exposition et circulation des objets précieux du Moyen Âge à nos jours, Mare et Martin, Paris, 2015.

[COQ 2003] COQUERY N., « Mode, commerce, innovation : la boutique parisienne au XVIIIe siècle. Aperçu sur les stratégies de séduction des marchands parisiens de luxe et de demi-luxe», dans L. HILAIRE-PÉREZ ET A.-F. GARÇON (dir.), Les chemins de la nouveauté. Innover, inventer au regard de l'histoire, Éditions du CTHS, Paris, 2003

[Cот 2007] Cотте M., Le choix de la révolution industrielle. Les entreprises de Marc Seguin et ses frères (18151835), Presses Universitaires de Rennes, Rennes, 2007.

[DAL 2009] Dalmasso A., «Vieilles et nouvelles technologies face aux mutations des années 1970. L'Isère et le bassin grenoblois (1974-1984)», dans LAMARD P., 1974-1984, une décennie de désindustrialisation ?, Éditions Picard, Paris, 2009.

[DAL, RoB 2007] Dalmasso A., ROBERT É., «Recherche et innovation dans l'industrie hydraulique : NeyrpicSogreah des années 1930 aux années 1960 », Annales historiques de l'électricité, p. 47-62, 2007.

[DAl 2001] Dalmasso A., «L'ingénieur, la Houille Blanche et les Alpes : une utopie modernisatrice ? , Le Monde alpin et rhodanien. Revue régionale d'ethnologie, $\mathrm{n}^{\circ} 1-3$, p. 25-38, 2001.

[DAU 2018] DaUMAS J.-C., La révolution matérielle, Une histoire de la consommation, France XIXe - XXe siècle, Flammarion, Paris, 2018.

[DRU 1993] DRUCKER P. F., Au-delà du capitalisme : La métamorphose de cette fin de siècle, Dunod, Paris, 1993. 
[FON 1984] FONTAINE L., Le voyage et la mémoire : colporteurs de l'Oisans au XIX siècle, Presses universitaires de Lyon, Lyon, 1984.

[Gal, Jud, Sai 2014] Gal S., Judet P. et SAINT MARTin J., « Histoire, innovation et territoires de montagne », dans AtTAli M., DAlmasso A., GRANET-ABISSET A.-M., Innovation en territoire de montagne : Le défi de l'approche interdisciplinaire, Presses Universitaire de Grenoble, Grenoble, 2014.

[Gal 2008] Galvez-Behar G., La République des inventeurs. Propriété et organisation de l'innovation en France (1791-1922), Presses Universitaires de Rennes, Rennes, 2008.

[GRI, FER 2007] GRISET P., FERnANDEZ A., «Les logiques spatiales de l'innovation, XIXe-XXe siècles », Histoire, économie \& société, $\mathrm{n}^{\circ} 2$, p. 3-13, 2007.

[Hil, Per 2017] Hilaire-Perez L., Perrin C. (modérateurs), Verna C., Galvez-Behar G., Le RouX M. et Rieu A.M. (intervenants), Rendez-vous de l'histoire Invention collective, communautés inventives, Blois, 7 octobre 2017.

[HIL 2009] HILAIRE-PereZ L., «L'invention technique et les figures de l'inventeur (XVIII ${ }^{\mathrm{e}}-\mathrm{XX}^{\mathrm{e}}$ siècles) Préface », Documents pour l'histoire des techniques, n¹7, p. 7-10, 2009.

[JAR 2009] JARRIGE F., «Le martyre de Jacquard ou le mythe de l'inventeur héroïque (France, XIX ${ }^{\mathrm{e}}$ siècle) », Tracés. Revue de Sciences humaines, ${ }^{\circ}$ 16, p. 99-117, 2009.

[JOB, TAL 2012] JOB L. et TALANDIER M., «Les dimensions économiques et territoriales des innovations : une application aux espaces de montagne. ", Regards croisés sur l'innovation en territoires de montagne, Grenoble, 2012.

[JOL 2020] JOLY H., « Profils et carrières d’élèves ingénieurs », Artefact, n¹3, p. 99-117, 2021.

[JOL 2003] JOLY H., «L'exploitation des actes de sociétés pour l'histoire des entreprises : intérêts et difficultés », Entreprises et Histoire, n³3, p. 120-126, 2003.

[JOL 1998] JOLY H., «Une source importante d'accès problématique pour l'historien: le premier registre du commerce (1920-1954)», Entreprises et histoire, n¹9, p. 159-164, 1998.

[KaA, Mon, Sim 2015] KaArtinen M., Montenach A. and Simonton D. (eds), Luxury and Gender in European Towns, 1700-1914, Routledge, New York, 2015.

[KIR, SMI 2004] KIRKUP G., SMITH Keller L., Inventing women: science, technology and gender, Polity press, the Open university, Oxford, 2004.

[LAM, Sto 2010] LAMARd P. et Stoskopf N., L’industrie chimique en question, Éditions Picard, Paris, 2010.

[LAN, 2015] LANOË C., «L'économie artisanale au prisme des actes de la pratique : le cas des gantiers-parfumeurs parisiens au XVIIIème siècle », Marché et organisations, n²4, p. 127-143, 2015.

[LE B en cours] LE BOT F., «Les laboratoires d'essais à l'articulation des écoles techniques supérieures, des entreprises, des territoires et de l'État, 1880-1960», HDR en cours, (garante : L. HILAIRE-PEREZ).

[LE B, Mic 2020] Le Bot F., Michel P. A., «Le travail des ingénieurs en entreprises », Artefact, n¹3, p. 9-46, 2020.

[LE B 2016] LE BOT F., «Enquête sur le céramiste en ingénieur. L’École de céramique de Sèvres instrument de la reconfiguration de l'industrie, 1870-1940», dans BOISARD P., DIDRY C., YOUNES D. (dir.), Les travailleurs de l'innovation. De l'entrepreneur aux salariés, Presses Universitaires de Rennes, Rennes, 2016.

[LE B, Per 2011] Le Bot F., PERrin C., « Mobiliser l'industrie de la chaussure, mobiliser ses territoires », Terrains \& travaux, $\mathrm{n}^{\circ} 19$, p. 205-224, 2011.

[LE B 2008] LE BOT F., « Confisquer les profits illicites : épuration économique ou simple mesure fiscale ? L'exemple des gantiers de Saint-Junien, Millau et Grenoble », dans BERGÈRE M. (dir.), L'épuration économique en France à la Libération, Presses Universitaires de Rennes, Rennes, 2008.

[LE B 2007] LE BOT F., La fabrique réactionnaire Antisémitisme, spoliations et corporatisme dans le cuir (19301950), Presses De Sciences Politiques, Paris, 2007.

[LE B 2005] LE BOT F., «La «famille» du cuir contre Bata: malthusianisme, corporatisme, xénophobie et antisémitisme dans le monde de la chaussure en France, 1930-1950 », Revue d'histoire moderne \& contemporaine, n $52-4$, p. 131-151, 2005.

[MAC 2003] MACLEOD C., «L'invention héroïque et la première historiographie de la révolution industrielle », dans HILAIRE-PEREZ L. ET GARÇON A.-F. (dir.), Les chemins de la nouveauté : innover, inventer au regard de l'histoire, Éditions CTHS, Paris, 2003. 
[MAT 2011] MATOUK J., «Le financement de l'innovation : une approche historique », Innovations, $\mathrm{n}^{\circ}$ 34, p. 123138, 2011.

[PER 2015] PERRIN C., « Le district en échec. L'exemple des tanneries de Château-Renault (v. 1860-1985) », dans COQUERY N., de OlIVEIRA M. (dir.), L'échec a-t-il des vertus économiques ? Comité pour l'histoire économique et financière de la France, Institut de la gestion publique et du développement économique, Paris, 2015.

[PER 2014] PERRIN C., «Le développement durable en perspective historique : l'exemple des tanneries », L'Homme \& la Société, n 193-194, p. 37-56, 2014.

[PON 2012] PONCIN P., Minitubes, un siècle d'histoire, APHID, Grenoble, 2012.

[ROB 2001] ROBERT E., L'ingénieur : moteur de l'innovation : un siècle de formation d'ingénieurs à Grenoble, MORET R. (dir.), Éditions des Vignes et l'éditeur, Grenoble, 2001.

[ROC 1997] ROCHE D., Histoire des choses banales. Naissance de la consommation XVIIe-XIXe siècle, Fayard, Paris, 1997.

[Roc 1991] ROCHE D., La culture des apparences, une histoire du vêtement XVII - XVIII siècle, Le Seuil, Paris, 1991.

[SCH 1999] SCHUMPETER J., Théorie de l'évolution économique. Recherches sur le profit, le crédit, l'intérêt et le cycle de la conjoncture, (réédition), Dalloz Éditions, Paris, 1999.

[SCH 1974] SCHUMPETER J., Capitalisme, socialisme et démocratie, Payot, Paris, 1974.

[STA 1993] STANLEY, A. Mothers and daughters of invention: notes for a revised history of technology, Lanham, Md. \& London, The Scarecrow press, inc., 1993.

[VER 1997] VERLEY P., La Révolution Industrielle, Éditions Gallimard, Paris, 1997.

[VER 1997] VERLEY P., L'échelle du monde. Essai sur l'industrialisation de l'Occident, Éditions Gallimard, Paris, 1997. 